\title{
ON LAKSHMIKANTHAM'S COMPARISON FOR ORDINARY DIFFERENTIAL EQUATIONS
}

\author{
GIOVANNI VIDOSSICH
}

ABSTRACT. The paper describes the relation between Lakshmikantham's comparison and other known facts.

V. Lakshmikantham introduced in [4] a new setting for the comparison of ordinary differential equations. The aim of this paper is to show that Lakshmikantham's comparison is a special case, in the sense explained by the statement of the theorem below, of the pairing comparison considered in Vidossich [6]. The implications of this discovery are: (i) all the results in the framework of Lakshmikantham's pairing can be generalized by setting them in the framework of the pairing comparison; (ii) the stability theorem Lakshmikantham and Leela [5, Theorem 2.13.1] claimed as new is in reality a special case of an older one [5, Theorem 2.13.3] which appeared in Conti and Sansone [2].

The above claims are consequences of the theorem below. Concerning (i), we avoid carrying out the program since the interested reader can do it easily; the proofs of the generalizations can be based on the existing arguments of the theorems to be generalized simply by substituting $\|\cdot\|$ by $\|\cdot\|^{2}$. We refer to Lakshmikantham and Leela [5], Ladas and Lakshmikantham [3] and Becker and Vidossich [1, Theorem 4] for the related results and bibliography.

Theorem. Let $X$ be a Banach space, $A \subseteq \mathbf{R} \times X, B \subseteq \mathbf{R}^{2}$ and $f, g: A \rightarrow X$, $\omega: B \rightarrow \mathbf{R}$ continuous functions. Let $O:] 0, \epsilon] \rightarrow \mathbf{R}$ be such that

$$
\lim _{h \downarrow 0} \frac{O(h)}{h}=0
$$

Then each one of the following statements,

$$
\begin{aligned}
\|x+h f(t, x)\| & \leq\|x\|+h \omega(t,\|x\|)+O(h), \\
\|x-y+h(f(t, x)-f(t, y))\| & \leq\|x-y\|+h \omega(t,\|x-y\|)+O(h), \\
\|x-y+h(f(t, x)-g(t, y))\| & \leq\|x-y\|+h \omega(t,\|x-y\|)+O(h),
\end{aligned}
$$

implies the corresponding one of the following statements,

$$
(f(t, x), x)_{-} \leq \omega(t,\|x\|)\left\|_{x}\right\|,
$$

Received by the editors September 4, 1974.

AMS (MOS) subject classifications (1970). Primary 34 A40.

Key words and phrases. Lakshmikantham comparison, pairing comparison, stability theorem. 


$$
\begin{aligned}
& (f(t, x)-f(t, y), x-y)_{-} \leq \omega(t,\|x-y\|)\|x-y\|, \\
& (f(t, x)-g(t, y), x-y)_{-} \leq \omega(t,\|x-y\| !\|x-y\|,
\end{aligned}
$$

but the converse fails. In other words, we have

$$
(i) \Rightarrow(i)^{*} \not(i) \quad(i=1,2,3) .
$$

In the above statement, $(\cdot, \cdot)$ _ denotes the generalized inner product on a Banach space $X$,

$$
(x, y)_{-}=\inf \{h(x) \mid h \in J(y)\}
$$

where $J: X \rightarrow 2^{*}$ is the duality map

$$
J(x)=\left\{h \in X^{*} \mid\|h\|=\|x\|, h(x)=\|x\|^{2}\right\} .
$$

When $X$ is a Hilbert space, $(\cdot, \cdot)$ _ coincides with the inner product.

Proof of Theorem. First we note the following property of the generalized pairing

$$
(x+y, x)_{-}=\|x\|^{2}+(y, x)_{-} .
$$

For, choosing $h \in J(x)$, we have

$$
h(x+y)=h(x)+h(y)=\|x\|^{2}+b(y)
$$

from which we have $(*)$ by taking inf $_{h}$.

$(1) \Rightarrow(1)^{*}$. From (1) it follows that

We have

$$
h \omega(t,\|x\|)+O(h) \geq\|x+h f(t, x)\|-\|x\| .
$$

$$
h \omega(t,\|x\|)\|x\|+O(h)\|x\| \geq\|x+h f(t, x)\|\|x\|-\|x\|^{2}
$$

(by the preceding inequality)

$$
\begin{aligned}
& \geq(x+h f(t, x), x)_{-}-\|x\|^{2} \\
& \quad \text { (by Cauchy-Schwartz } \\
& \geq\|x\|^{2}+(h f(t, x), x)_{-}-\|x\|^{2} \quad(\text { by }(*)) \\
& =h(f(t, x), x)_{-} .
\end{aligned}
$$

(by Cauchy-Schwartz inequality)

Dividing both members of this inequality by $h>0$ and taking $\lim _{h \downarrow 0}$ we get $(1)^{*}$.

$$
(2) \Rightarrow(2)^{*} \text { and }(3) \Rightarrow(3)^{*} \text { can be proved as }(1) \Rightarrow(1)^{*} \text {. The other part of }
$$
the Theorem will be proved by exhibiting counterexamples in the real line.

$(1)^{*} \not(1)$. Let $X=\mathbf{R}$ and define $\left.\left.f, \omega: \mathbf{R} \times\right] 0,1\right] \rightarrow \mathbf{R}$ by

$$
f(t, x)=\sin x^{-1}=\omega(t, x) \text {. }
$$

Then $(1)^{*}$ holds for $f, \omega$. Assume (1) holds for some function $O$ with $\lim _{h \backslash 0}(O(h) / h)=0$ and argue for a contradiction. Fix $\left.\left.h \in\right] 0,1\right]$. There is a sequence $\left(x_{h, n}\right)_{n}$ in $\left.] 0, h\right]$ such that $\lim _{n} x_{h, n}=0$ and $\sin \left(1 / x_{h, n}\right)=-1$. We have 


$$
\begin{aligned}
\left|x_{h, n}+h f\left(t, x_{h, n}\right)\right| & =h-x_{h, n}, \\
\left|x_{h, n}\right|+h \omega\left(t,\left|x_{h, n}\right|\right)+O(h) & =x_{h, n}-h+O(h) .
\end{aligned}
$$

Therefore from the assumed (1) we have

$$
h-x_{h, n} \leq x_{h, n}-h+O(h) .
$$

Taking $\lim _{n}$ we get $h \leq-h+O(h)$ which implies $O(h) / h \geq 2$ (all $\left.h\right)$, a contradiction.

$(2)^{*} \not(2)$. Let $X=\mathbf{R}$ and define $f: \mathbf{R}^{+} \times \mathbf{R} \rightarrow \mathbf{R}, \omega: \mathbf{R}^{2} \rightarrow \mathbf{R}$ by

$$
f(t, x)=-t x, \quad \omega(t, x)=0 .
$$

Since $f(t, \cdot)$ is decreasing, (2) holds for $f, \omega$. Assume (2) holds for some function $O$ with $\lim _{h \backslash 0}(O(h) / h)=0$, and argue for a contradiction. Fix $h>0$. There are sequences $\left(x_{h, n}\right),\left(y_{h, n}\right)_{n}$ of real numbers such that

$$
0<x_{h, n}-y_{h, n}<h \text { and } \lim _{n}\left(x_{h, n}-y_{h, n}\right)=0 \text {. }
$$

Let $t_{h, n}=1 /\left(x_{h, n}-y_{h, n}\right)$. We have

$$
\begin{aligned}
& \left|x_{h, n}-y_{h, n}+h\left(f\left(t_{h, n}, x_{h, n}\right)-f\left(t_{h, n}, y_{h, n}\right)\right)\right|=h-\left(x_{h, n}-y_{h, n}\right), \\
& \left|x_{h, n}-y_{h, n}\right|+h \omega\left(t_{h, n},\left|x_{h, n}-y_{h, n}\right|\right)+O(h)=x_{h, n}-y_{h, n}+O(h) .
\end{aligned}
$$

Therefore from the assumed (2) we have

$$
h-\left(x_{h, n}-y_{h, n}\right) \leq x_{h, n}-y_{h, n}+O(h) .
$$

Taking $\lim _{n}$ we get $h \leq O(h)$ which implies $O(h) / h \geq 1$ (all $h$ ), a contradiction.

$(3)^{*} \neq(3)$. This follows by taking $g=f$ in the above example used to show $(2)^{*} \neq(2)$. q.e.d.

\section{REFERENCES}

1. R. Becker and G. Vidossich, Some applications of a simple criterion for the existence of periodic solutions of ordinary differential equations, J. Math. Anal. Appl. (to appear).

2. R. Conti and G. Sansone, Equazioni differenziali nonlineari, Edizioni Cremonese, Rome, 1956; English transl., Internat. Ser. of Monographs in Pure and Appl. Math., vol. 67, Macmillan, New York, 1964. MR 19, 547; 31 \#1417.

3. G. E. Ladas and V. Lakshmikantham, Differential equations in abstract spaces, Academic Press, New York, 1972.

4. V. Lakshmikantham, Notes on a variety of problems of differential systems, Arch. Rational Mech. Anal. 10 (1962), 119-126. MR 25 \#5237.

5. V. Lakshmikanthain and S. Leela, Differential and integral inequalities, vols. I, II, Academic Press, New York, 1969.

6. G. Vidossich, Existence, comparison and asymptotic behavior of solutions of ordinary differential equations in finite and infinite dimensional Banach spaces, Notas de Matemática, no.24, Universidade de Brasília, 1972.

DEPARTMENT O DE MATEMATICA, UNIVERSIDADE DE BRASILIA, 70.000 BRASILIA D.F., BRASIL 